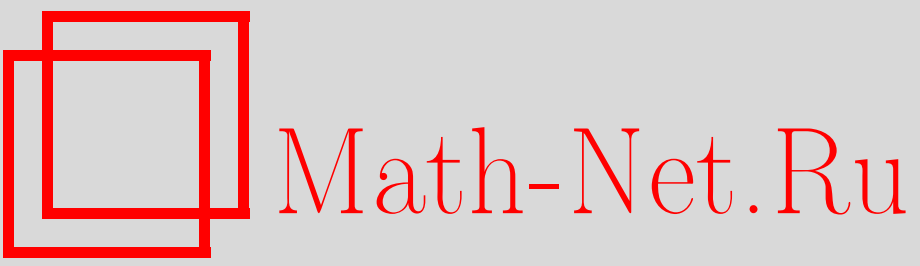

В. Н. Сорокин, А. С. Вшивцев, Н. В. Норин, Решение спектральной задачи для уравнения Шредингера с вырожденным полиномиальным потенциалом четной степени, ТМФ, 1996, том 109, номер 1, 107-123

DOI: https://doi.org/10.4213/tmf1215

Использование Общероссийского математического портала Math-Net.Ru подразумевает, что вы прочитали и согласны с пользовательским соглашением

http://www. mathnet.ru/rus/agreement

Параметры загрузки:

IP: 34.229 .45 .116

26 апреля 2023 г., 15:16:13 


\author{
ТЕОРЕТИЧЕСКАЯ \\ И МАТЕМАТИЧЕСКАЯ \\ ФИЗИКА \\ Том 109, № 1 \\ октябрь, 1996
}

А. С. Вшивцев* , Н. В. Норин*, В.Н. Сорокин ${ }^{\dagger}$

\title{
РЕШЕНИЕ СПЕКТРАЛЬНОЙ ЗАДАЧИ ДЛЯ УРАВНЕНИЯ ШРЕДИНГЕРА С ВЫРОЖДЕННЫМ ПОЛИНОМИАЛЬНЫМ ПОТЕНЦИАЛОМ ЧЕТНОЙ СТЕПЕНИ
}

Для стационарного уравнения Шредингера с вырожденным типом потенциалов $U(x)=x^{2 r}$, где $r \in Z_{+}$, описываюшим ситуации, связанные с фазовыми переходами в квантовых системах, вскрыта симметрия задачи путем приведения ее к алгебраической для нахождения спектра. Построена аналитическая процедура сходящейся теории возмущений нахождения собственных значений для потенциалов указанного типа, которая реализована численно при $r=2,3, \ldots, 18$. Найдены нижние уровни энергии и описаны их свойства.

\section{ВВЕДЕНИЕ}

Нахождение спектра и построение сходяшегося ряда теории возмушений для ангармонического осциллятора - одна из проблем. Эта задача квантовой механики, сформулированная в работах Шредингера и Фока $[1,2]$, не потеряла своей актуальности и по настоящее время. Несмотря на то что ее изучению отводится почетное место в любом учебнике по квантовой механике, а также посвящено значительное число статей, включаюших как некоторые эвристические рассуждения, так и строгие математические результаты, тема еще далека от полного понимания. Интерес к этой теме обусловлен рядом причин. Одна из них - неудовлетворенность, с которой теоретики обращаются с расходящимися рядами теории возмущений, а также неумение в полной степени осознать их поведение и, что наиболее важно, аналитическую структуру по константе связи и как следствие утерю тех или иных физических особенностей при решении конкретных задач. В самом деле, основная часть достижений современной квантовой теории поля основана на результатах обычной теории возмушений, ряды которой в большинстве случаев асимптотические. По мере того как этот факт осознается, у исследователей возникает интерес к формулировке различных непертурбативных методов. Так,

\footnotetext{
* Московский государственный институт радиотехники, электроники и автоматики (технический университет)

${ }^{\dagger}$ Московский государственный университет
} 
например, одномерным аналогом полевых теорий с потенциалом типа $\phi^{4}$ является ангармонический осциллятор.

В данной работе мы рассмотрим решение уравнения

$$
\left\{\frac{\partial^{2}}{\partial x^{2}}-x^{2 r}\right\} \phi(x)=E \phi(x) .
$$

Решение задачи нахождения спектра и волновых функций при помоши вскрытия симметрии уравнения сводится к алгебраической задаче. Реализация этой процедуры состоит в выполнении следуюших этапов:

1. Изучение асимптотического поведения $(x \rightarrow \infty)$ решений уравнения $-\phi(x) \sim$ $e^{-\frac{x^{r+1}}{r+1}}$ называемого метода квазиточно решаемых задач [3-8].

2. Далее с помощью методов теории функций (аналогично тому, как это реализовали Лаггер, Эрмит и т.д.) с весами $e^{-x^{r+1}}$ строятся так называемые биортогональные многочлены $\Lambda_{n}^{p}(x)[9,10]$ :

$$
\Lambda_{n}^{p}(x)=e^{x^{r}}\left\{\frac{\partial}{\partial x}\right\}^{n} x^{n+p} \frac{e^{-x^{r}}}{n !}
$$

Этот шаг позволяет правильно описывать возбужденные состояния спектра.

3. На третьем этапе на основе знания структуры решения рассматриваемого уравнения (аналогично тому, как это имеет место при решении обыкновенных дифференциальных уравнений с постоянными коэффициентами при использовании метода интегральных преобразований с рядом Лапласа $e^{-p x}$ ) задача сводится к алгебраической.

Мы не будем описывать различные подходы к решению уравнения Шредингера, чтобы не загромождать данную работу. Если все же у читателя имеется соответствуюший интерес, то по этим вопросам опубликован обширный материал [11-16]. Отметим лиш тот факт, что наш подход ранее в литературе не обсуждался.

Более практический (физический) интерес к обсуждению решения уравнения Шредингера с потенциалом $x^{2 r}$, не связанньй с построением формальной теории, обусловливается следующими причинами. При реализации процедуры разделения переменных релятивистские уравнения Дирака и Клейна-Гордона-Фока в ряде случаев (после преобразований систем координат) могут быть приведены к такому виду $[17,18]$.

Следуюший аспект представляется нам более важным с точки зрения приложений к физическим задачам. Рассмотрение потенциалов типа $x^{2 r}$ соответствует критической ситуации обрашения в нуль всех коэффициентов полинома $P_{2 r}(x)$, кроме старшего. Эта ситуация отвечает вырождению и обычно связывается с фазовыми переходами в системе [19-21]. Так, рассмотрим тривиальный пример с таким типом потенциала: $x^{4}+\varepsilon x^{2}=P_{4}^{+}(x), \varepsilon>0$. Этот потенциал достаточно гладкий, выпуклый вниз, и для него выполнены все условия процедуры ВКБ-приближения $[14,16]$. В точке $\varepsilon=0$, соответствуюшей “началу” фазового перехода, имеется вырождение (при $x_{0}=0$ ) и, как 
известно (см., например, $[19,22,23])$, должна происходить перестройка спектра. Затем потенциал изменяет симметрию $P_{4}^{-}(x)=x^{4}-\varepsilon x^{2}, \varepsilon>0$, и соответственно могут происходить изменения физических свойств системы, определяемые спектром. Таким образом, точка вырождения (при управляюшем параметре $\varepsilon=0$, вообше говоря, зависяшем от внешних параметров задачи) должна быть хорошо изучена. Возможно, что знание тонких спектральных свойств исследуемой задачи позволит точнее сформулировать дополнительные критерии фазовых переходов в реальных физических системах. Это оказывается тем более важным фактом, что спектральные задачи, рассмотренные ранее $[14,16]$, касались асимптотических свойств спектра изучаемого уравнения без подробного исследования поведения нижних уровней. Наше рассмотрение позволяет ликвидировать этот пробел в теории уравнения Шредингера для потенциалов такого типа.

Данная статья организована следующим образом. В разделе 1 описана процедура сведения решения исследуемого уравнения Шредингера к алгебраической задаче. В разделе 2 обсужден ангармонический осциллятор с потенциалом вида $\left(x^{2}-\varepsilon\right)^{2}$. В разделе 3 представлены численные результаты решения полученной алгебраической задачи при $r=2,3, \ldots, 18$ и дано их обсуждение.

\section{1. АНГАРМОНИЧЕСКИЙ ОСЦИЛЛЯТОР С ВЫРОЖДЕНИЕМ}

Перейдем теперь к ангармоническому осциллятору. Зафиксируем натуральное число $r$ и поставим следуюшую задачу.

Требуется найти все значения параметра $\lambda$, при каждом из которых дифференциальное уравнение

$$
\phi^{\prime \prime}-x^{2 r} \phi=-\lambda \phi
$$

имеет решение $\phi(x)$ из пространства $L_{2}(R)$, т.е.

$$
\int_{-\infty}^{+\infty}|\phi(x)|^{2} d x<+\infty
$$

В частности, при $r=1$ получим задачу о гармоническом осцилляторе.

Найдем асимптотику решений уравнения (1) при $x \rightarrow \pm \infty$. Записывая волновую функцию в виде $\phi(x)=\exp s(x)$, приходим к уравнению

$$
s^{\prime \prime}+s^{2}-x^{2 r}=-\lambda .
$$

При больших $x$ решения этого уравнения эквивалентны решениям уравнения $s^{2}=x^{2 r}$, т.е. функциям $s(x)= \pm|x|^{r+1} /(r+1)$. Таким образом, при любом значении параметра $\lambda$ общее решение уравнения (1) имеет вид

$$
\phi(x)=A \exp \left\{\frac{|x|^{r+1}}{r+1}(1+o(1))\right\}+B \exp \left\{-\frac{|x|^{r+1}}{r+1}(1+o(1))\right\}, \quad x \rightarrow \pm \infty
$$


где $A$ и $B$-произвольные постоянные. Поэтому условие (2) можно заменить граничными условиями

$$
\phi(x) \rightarrow 0 \text { при } x \rightarrow \pm \infty
$$

или условиями

$$
\phi(x)=\exp \left\{-\frac{|x|^{r+1}}{r+1}(1+o(1))\right\} \text { при } x \rightarrow \pm \infty .
$$

Дифференциальное выражение $-\phi^{\prime \prime}+x^{2 r} \phi$ определяет положительный неограниченный самосопряженный оператор в гильбертовом пространстве $L_{2}(R)$, который тем самым имеет положительный дискретный спектр (заметим, что спектр (1) является невырожденным),

$$
0<\lambda_{0}<\lambda_{1}<\cdots<\lambda_{n}<\cdots, \quad \lambda_{n} \rightarrow \infty
$$

Обозначим через

$$
N(\lambda)=\sum_{\lambda_{n}<\lambda} 1
$$

функцию распределения собственных значений этого оператора. По теореме Титчмарша-Карлемана о распределении собственных значений полуограниченных операторов Штурма-Лиувилля имеем следуюшую асимптотическую формулу:

$$
N(\lambda) \sim \frac{1}{\pi} \int_{x^{2 r}<\lambda} \sqrt{\lambda-x^{2 r}} d x, \quad \lambda \rightarrow \infty
$$

Вычисляя интеграл (3), получим

$$
N(\lambda) \sim \lambda^{(r+1) / 2 r} B(3 / 2,1 / 2 r) / \pi r, \quad \lambda \rightarrow \infty .
$$

$\mathrm{K}$ решению задачи нахождения спектра ангармонического осциллятора при $r>1$ можно применить метод, основанный на ее связи с многочленами совместной ортогональности, которые описывались нами ранее [10].

Воспользуемся явной симметрией задачи относительно преобразования $x \rightarrow-x$ и будем вначале искать все четные решения уравнения (1), которые удовлетворяют начальному условию $\phi(0, \lambda)=1$. Напишем разложение волновой функции в ряд Тейлора

$$
\phi(x, \lambda)=\sum_{p=0}^{\infty} a_{p}(\lambda) x^{2 p} .
$$

Подстановка ряда (5) в уравнение (1) приводит к рекуррентному соотношению

$$
(2 p+2)(2 p+1) a_{p+1}-a_{p-r}=-\lambda a_{p}, \quad p=0,1,2, \ldots,
$$


с начальными условиями

$$
a_{-r}=\ldots=a_{-1}=0, \quad a_{0}=1 .
$$

Таким образом, мы пришли к многочленам совместной ортогональности с матрицей Якоби, соответствуюшей (6a). Для того чтобы в этом убедиться, достаточно положить $b_{p}(\lambda)=\eta^{p} a_{p}(-\lambda / \eta)$, где $\eta=\exp (\pi i /(r+1))$. Действительно, при этом преобразовании рекуррентное соотношение $(6 \mathrm{a})$ перейдет в соотношение

$$
(2 p+2)(2 p+1) b_{p}(\lambda)+b_{p-r}(\lambda)=\lambda b_{p}(\lambda), \quad p=0,1,2, \ldots .
$$

Другими словами, спектральные функции оператора (6) определены на луче $\Delta=\{t \eta$ : $t>0\}$. Многочлены $a_{p}(\lambda)$ инвариантны относительно группы $Z_{r+1}$.

Примем нормировку, при которой матрица Якоби станет почти симметричной, а именно, положим

$$
q_{p}(\lambda)=k_{p} a_{p}(\lambda)
$$

где

$$
k_{p}=(r+1)^{\frac{2 p}{r+1}} \Gamma\left(\frac{2 p+r}{r+1}\right) / \Gamma\left(\frac{r}{r+1}\right), \quad p=0,1,2, \ldots
$$

Тогда многочлены $q_{p}(\lambda)$ будут удовлетворять рекуррентному соотношению

$$
\begin{gathered}
(p+1) q_{p+1}-(p-r / 2) q_{p-r}=-\frac{\lambda}{2(r+1)^{\frac{r-1}{r+1}} \Gamma\left(\frac{r-1}{r+1}\right)} B\left(\frac{2 p+1}{r+1}, \frac{r-1}{r+1}\right) q_{p}, \\
p=0,1,2, \ldots, \quad q_{-r}=\cdots=q_{-1}=0, \quad q_{0}=1,
\end{gathered}
$$

которому соответствует нормированная матрица Якоби

$$
\left(\begin{array}{ccccccc}
0 & \ldots & 0 & \beta_{0} & & & \\
\alpha_{0} & 0 & \ldots & 0 & \beta_{1} & & \\
& \alpha_{1} & 0 & \ldots & 0 & \beta_{2} & \\
& & \ddots & \ddots & & \ddots & \ddots
\end{array}\right),
$$

где

$$
\begin{aligned}
\alpha_{p} & =\frac{2(p+1)(r+1)^{\frac{r-1}{r+1}} \Gamma\left(\frac{r-1}{r+1}\right)}{B\left(\frac{2 p+1}{r+1}, \frac{r-1}{r+1}\right)}, \\
\beta_{p} & =-\frac{2(p+r / 2)(r+1)^{\frac{r-1}{r+1}} \Gamma\left(\frac{r-1}{r+1}\right)}{B\left(\frac{2 p+1}{r+1}, \frac{r-1}{r+1}\right)}
\end{aligned}
$$


и $p=0,1,2, \ldots$. Начиная с этого момента считаем, что $r>1$.

Из формул (7), (7a), (7б) и формулы Стирлинга вытекает, что элементы нормированной матрицы Якоби имеют следуюшую асимптотику:

$$
\alpha_{p} \sim(2 p)^{\frac{2 r}{r+1}}, \quad \beta_{p} \sim(2 p)^{\frac{2 r}{r+1}}, \quad p \rightarrow \infty
$$

Однако тогда в соответствии с теорией потенциала Гончара-Рахманова мы можем сделать вывод, что спектральные функции оператора (7) асимптотически ведут себя как

$$
\cos ^{-1}\left(d \lambda^{\mu}\right), \quad|\lambda| \rightarrow \infty
$$

где $d=B(1 / 2 r, 1 / 2) /(2 r+2), \mu=(r+1) / 2 r$. Положительные полюсы функции $(8)$ равны (сравни с результатом, полученным методом ВКБ)

$$
\lambda_{n}=\{\pi r(2 n+1) / B(3 / 2,1 / 2 r)\}^{2 r /(r+1)}, \quad n=0,1,2, \ldots,
$$

что согласуется с формулой (4). Напомним, что мы пока изучаем лишш четную часть спектра.

Рассмотрим производящую функцию многочленов $q_{p}(\lambda)$ :

$$
\Phi(\lambda, w)=\sum_{p=0}^{\infty} q_{p}(\lambda) w^{\frac{2 p}{r+1}}
$$

Умножим обе части равенства $(7)$ на $w^{\frac{2 p+1-r}{r+1}}$ и просуммируем по всем $p=0,1,2, \ldots$. Имеем

$$
\sum_{p=0}^{\infty}(p-r / 2) q_{p-r} w^{\frac{2 p+1-r}{r+1}}=\frac{r+1}{2} w^{\frac{r+2}{r+1}} \frac{\partial}{\partial w} w^{\frac{r}{r+1}} \Phi(\lambda, w) .
$$

Таким образом, в левой части равенства мы получим следующий дифференциальный оператор:

$$
\frac{r+1}{2}\left\{\left(1-w^{2}\right) \frac{\partial}{\partial w} \Phi(\lambda, w)-\frac{r}{r+1} w \Phi(\lambda, w)\right\}
$$

Записывая интегральное представление бета-функции, получим

$$
\sum_{p=0}^{\infty} B\left(\frac{2 p+1}{r+1}, \frac{r-1}{r+1}\right) q_{p}(\lambda) w^{\frac{2 p+1-r}{r+1}}=w^{-\frac{r-1}{r+1}} \int_{0}^{1}(1-t)^{-\frac{2}{r+1}} t^{-\frac{r}{r+1}} \Phi(t, w) d t
$$

Обозначим

$$
\tilde{\lambda}=\lambda(r+1)^{-2 r /(r+1)} / \Gamma\left(\frac{r-1}{r+1}\right) .
$$


Окончательно приходим к выводу, что производяшая функция $\Phi(w)=\Phi(\lambda, w)$ является решением следуюшего интегродифференциального уравнения:

$$
\left(1-w^{2}\right) \Phi^{\prime}-\frac{r}{r+1} w \Phi=-\tilde{\lambda} w^{-\frac{r-1}{r+1}} \Phi^{*},
$$

где $\Phi^{*}(w)$ - результат применения к $\Phi(w)$ интегрального оператора

$$
\Phi^{*}(w)=\int_{0}^{1} t^{-\frac{r}{r+1}}(1-t)^{-\frac{2}{r+1}} \Phi(t w) d t
$$

при этом должно выполняться начальное условие $\Phi(0)=1$. По сушеству, функция $\Phi(w)=\Phi(\lambda, w)$ получается применением к волновой функции $\phi(x)=\phi(x, \lambda)$ следующего обобщенного преобразования Лапласа:

$$
\phi(x) \rightleftharpoons \Phi(w)=\frac{1}{\Gamma\left(\frac{r}{r+1}\right)} \sqrt[r+1]{\frac{r+1}{w^{r}}} \int_{0}^{+\infty} \phi(x) \exp \left\{-\frac{x^{r+1}}{r+1} \frac{1}{w}\right\} x^{r-1} d x
$$

действительно,

$$
x^{2 p} \rightleftharpoons \frac{1}{\Gamma\left(\frac{r}{r+1}\right)} \sqrt[r+1]{\frac{r+1}{w^{r}}} \int_{0}^{+\infty} x^{2 p+r-1} \exp \left\{-\frac{x^{r+1}}{r+1} \frac{1}{w}\right\} d x=k_{p} w^{\frac{2 p}{r+1}}
$$

Следующее обобшенное преобразование Миттаг-Лефиера будет обратным к (10):

$$
\phi(x)=\frac{1}{2 \pi i} \int_{|w|=\rho} \Phi\left(w^{\frac{r+1}{2}}\right) E\left(\frac{x^{2}}{w}\right) \frac{d w}{w},
$$

где $0<\rho<1$, и $E(z)=\sum_{p=0}^{\infty} z^{p} / k_{p}$ - функция Миттаг-Леф̆лера.

Отсюда мы можем сделать важный вывод, что граничные условия (2б) выполняются в том и только в том случае, если $w=1$ - правильная точка функции $\Phi(w)$, и, значит, эта функция регулярна в правой полуплоскости $\Re w>0$.

Этим замечанием, по существу, обусловливается выбор производяшей функции в форме, предложенной выше. Конечно, мы могли бы с самого начала решать уравнение ангармонического осциллятора (1) с помошью интегрального преобразования (10). Формально этот метод полностью эквивалентен предложенному нами методу рекуррентных соотношений, который мы тем не менее считаем здесь более удачным, поскольку, как было отмечено выше, известные в теории функций результаты о матрицах Якоби позволяют сразу же получить асимптотику спектра и асимптотику спектральной меры дискретного оператора Штурма-Лиувилля (7). Последнее обстоятельство также может быть использовано при построении эффективного численного метода, отличающегося от описанного в настоящей работе. 
При $\tilde{\lambda}=0$ уравнение (9а) обрашается в дифференциальное уравнение первого порядка

$$
\left(1-w^{2}\right) \Phi^{\prime}-\frac{r}{r+1} w \Phi=0
$$

Решая его методом разделения переменных и учитывая начальное условие, получим

$$
\Phi(w)=\left(1-w^{2}\right)^{-\frac{r}{2(r+1)}} .
$$

Поэтому решения интегродифференщиального уравнения (9a) удобно искать в виде

$$
\Phi(w)=H(w)\left(1-w^{2}\right)^{-\frac{r}{2(r+1)}} .
$$

Подстановка формулы (11) в уравнение (9а) приводит к уравнению

$$
\left(1-w^{2}\right) H^{\prime}=\tilde{\lambda} w^{-\frac{r-1}{r+1}}\left(1-w^{2}\right)^{\frac{r}{2(r+1)}} \int_{0}^{1} t^{-\frac{r}{r+1}}(1-t)^{-\frac{2}{r+1}}\left(1-t^{2} w^{2}\right)^{-\frac{r}{2(r+1)}} H(t w) d t
$$

Имея в виду дальнейшую алгебраизацию задачи, сделаем в уравнении (12) дробно-линейную замену переменной

$$
x=(1-w) /(1+w),
$$

при которой правая полуплоскость $\Re w>0$ переходит в единичный круг $|x|<1$, и введем новую неизвестную функцию по формуле

$$
\tilde{\Lambda}(x)=H(w) .
$$

Тогда левая часть уравнения (12) примет вид

$$
\left(1-\left(\frac{1-x}{1+x}\right)^{2}\right) \cdot \tilde{\Lambda}^{\prime}(x) \cdot\left(-\frac{2}{(1+x)^{2}}\right)^{-1}=-2 x \tilde{\Lambda}^{\prime}(x)
$$

Прежде чем сделать замену (13) в правой части уравнения (12), введем новую переменную интегрирования $\theta=t w$. Тогда справа получим

$$
-\tilde{\lambda} w^{\frac{2-r}{r+1}}\left(1-w^{2}\right)^{\frac{r}{2(r+1)}} \int_{0}^{w} \theta^{-\frac{r}{r+1}}(w-\theta)^{-\frac{2}{r+1}}\left(1-\theta^{2}\right)^{-\frac{r}{2(r+1)}} H(\theta) d \theta .
$$

Теперь в (15) можно сделать замену (13) и одновременно аналогичную замену переменной интегрирования $u=(1-\theta) /(1+\theta)$. В результате $(15)$ примет вид

$$
-\tilde{\lambda} 2^{-\frac{2}{r+1}}(1-x)^{\frac{2-r}{r+1}} x^{\frac{r}{2(r+1)}} \int_{x}^{1}(1-x / u)^{-\frac{2}{r+1}}(1-u)^{-\frac{r}{r+1}} u^{\frac{r-2}{2(r+1)}} \tilde{\Lambda}(u) \frac{d u}{u} .
$$


И, наконец, положим $\nu=\tilde{\lambda} \cdot 2^{-\frac{2}{r+1}}$. Тем самым мы показали, что функция $\tilde{\Lambda}(x)$ удовлетворяет следующему интегродифференциальному уравнению:

$$
x^{\frac{r+2}{2(r+1)}}(1-x)^{\frac{r-2}{r+1}} \tilde{\Lambda}^{\prime}(x)=\nu \int_{x}^{1}(1-x / u)^{-\frac{2}{r+1}}(1-u)^{-\frac{r}{r+1}} u^{\frac{r-2}{2(r+1)}} \tilde{\Lambda}(u) \frac{d u}{u} .
$$

Начальное условие перейдет в начальное условие $\tilde{\Lambda}(1)=1$. Напомним, что спектральное условие на функцию $\Phi(w)$ заключалось в том, что эта функция должна быть регулярной в правой полуплоскости $\Re w>0$. Учитывая (11) и (14), мы можем сформулировать спектральное условие на функцию $\tilde{\Lambda}(x)$, а именно, функция

$$
\tilde{\Lambda}(x) x^{-\frac{r}{2(r+1)}}=(1-x)^{\frac{3}{r+1}} \Lambda(x)
$$

должна быть регулярной в единичном круге $|x|<1$.

В результате уравнение (16) преобразуется в уравнение

$$
x(1-x) \Lambda^{\prime}(x)+\frac{r-(r+6) x}{2(r+1)} \Lambda(x)=\nu \int_{x}^{1}(u-x)^{-\frac{2}{r+1}}(1-u)^{\frac{3-r}{r+1}} \Lambda(u) d u .
$$

Отметим, что в левой части уравнения (17) стоит дифференциальный оператор гипергеометрического типа, а в правой части - интеграл типа свертки Меллина.

Спектральная задача ставится теперь следуюшим образом. Требуется найти все значения параметра $\nu$, при каждом из которых уравнение (17) имеет своим решением не равную тождественно нулю функцию $\Lambda(x)$, регулярную в единичном круге.

Алгебраизация задачи. Сведем задачу (17) к алгебраической. Будем искать решение этого уравнения в виде степенного ряда

$$
\Lambda(x)=\sum_{n=0}^{\infty} c_{n} x^{n}
$$

Подстановка ряда (18) в левую часть уравнения (17) даст степенной ряд

$$
\sum_{n=0}^{\infty}\left[c_{n}\left(n+\frac{r}{2(r+1)}\right)-c_{n-1}\left(n+\frac{4-r}{2(r+1)}\right)\right] x^{n} .
$$

Для того чтобы найти разложение в степенной ряд правой части уравнения (17), обозначим

$$
J(x)=\int_{x}^{1}(u-x)^{-\frac{2}{r+1}}(1-u)^{\frac{3-r}{r+1}} \Lambda(u) d u
$$


и вычислим преобразование Меллина этой функции. Получим $J(x)=\sum_{n=0}^{\infty} R_{n} x^{n}$, где $R_{n}=\sum_{m=0}^{\infty} c_{m} \rho_{n, m}$. Здесь

$$
\begin{aligned}
& \rho_{n, m}= \frac{\left(\frac{2}{r+1}\right)_{n}}{n !} B\left(\frac{r-1}{r+1}, \frac{4}{r+1}\right) b_{m-n}, \\
& b_{k}= \begin{cases}\left(\frac{r-1}{r+1}\right)_{k} /\left(\frac{r+3}{r+1}\right)_{k}, & \text { если } k>0, \\
1, & \text { если } k=0, \\
\left(-\frac{2}{r+1}\right)_{|k|} /\left(\frac{2}{r+1}\right)_{|k|}, & \text { если } k<0 .\end{cases}
\end{aligned}
$$

При этом используется обозначение $(c)_{n}=c(c+1) \ldots(c+n-1)$ для символа Похгаммера.

Таким образом, учитывая (19) и вводя обозначение

$$
\varkappa=\left\{\nu B\left(\frac{r-1}{r+1}, \frac{4}{r+1}\right)\right\}^{-1},
$$

приходим к следующей задаче на собственные числа:

$$
\sum_{m=0}^{\infty} b_{m-n} c_{m}=\varkappa \frac{n !}{\left(\frac{2}{r+1}\right)_{n}}\left[c_{n}\left(n+\frac{r}{2(r+1)}\right)-c_{n-1}\left(n+\frac{4-r}{2(r+1)}\right)\right] .
$$

Можно считать последовательность $\overline{\bar{c}}=\left(c_{0}, c_{1}, c_{2}, \ldots\right)$ элементом гильбертова пространства $l_{2}$. Тогда соотношение (20) будет иметь вид $B \overline{\bar{c}}=x A \overline{\bar{c}}$, где $A$ и $B$ - операторы, определяемые левой и правой частями соотношения (20), соответственно. Полученное уравнение также можно записать в виде $K \overline{\bar{c}}=x \overline{\bar{c}}$, где $K=A^{-1} B$. Нетрудно убедиться в том, что $K$ - компактный оператор. Таким образом, соотношение (20) представляет собой задачу на собственные числа некоторого компактного оператора в гильбертовом пространстве $l_{2}$. Заметим, что спектр полученного компактного оператора состоит из чисел $C / \lambda_{n}$, где $\lambda_{n}$ - собственные значения исходного уравнения Шредингера, а $C$ некоторая постоянная.

Методы приближенного вычисления собственных значений таких операторов хорошо известны. Мы покажем, что даже простейший вариант приближения компактного оператора (20) конечномерными позволяет получить большую информацию о спектрах ангармонических осцилляторов, хотя при проведении расчетов можно существенно улучшить скорость сходимости методом Рунге и другими численными методами.

Аналогичным образом будем искать нечетную часть спектра ангармонических осцилляторов.

Пусть $\phi(x, \lambda)$ - нечетные решения уравнения $(41)$, удовлетворяюшие начальному условию $\phi_{x}^{\prime}(0, \lambda)=1$. Напишем разложение этой функции в ряд Тейлора

$$
\phi(x, \lambda)=\sum_{p=0}^{\infty} a_{p}(\lambda) x^{2 p+1}
$$


подстановка которого в уравнение (1) приводит к рекуррентному соотношению

$$
(2 p+3)(2 p+2) a_{p+1}-a_{p-r}=-\lambda a_{p}, \quad p=0,1,2, \ldots,
$$

с начальными условиями

$$
a_{-r}=\cdots=a_{-1}=0, \quad a_{0}=1 .
$$

Таким образом, мы пришли к многочленам совместной ортогональности. Применим нормировку

$$
q_{p}(\lambda)=k_{p} a_{p}(\lambda), \quad k_{p}=(2 r+1)^{\frac{2 p}{r+1}+1} \Gamma\left(\frac{2 p+r+2}{r+1}\right) / \Gamma\left(\frac{1}{r+1}\right) .
$$

Тогда многочлены $q_{p}(\lambda)$ будут удовлетворять рекуррентному соотношению

$$
\begin{gathered}
(p+1) q_{p+1}-\left(p-\frac{r-2}{2}\right) q_{p-r}=-\frac{\lambda}{2(r+1)^{\frac{r-1}{r+1}} \Gamma\left(\frac{r-1}{r+1}\right)} B\left(\frac{2 p+3}{r+1}, \frac{r-1}{r+1}\right) q_{p}, \\
q_{-r}=\cdots=q_{-1}=0, \quad q_{0}=1 .
\end{gathered}
$$

Из (21) получим, что производяшая функция многочленов $q_{p}(\lambda)$ удовлетворяет интегродифференциальному уравнению

$$
\left(1-w^{2}\right) \Phi^{\prime}-\frac{r+2}{r+1} w \Phi=-\tilde{\lambda} w^{\frac{1-r}{1+r}} \Phi^{*}(w)
$$

где

$$
\Phi^{*}(w)=\int_{0}^{1} t^{\frac{2-r}{r+1}}(1-t)^{-\frac{2}{r+1}} \Phi(t w) d t
$$

при этом выполняется начальное условие $\Phi(0)=1$.

Функция $\Phi(w)$ получается из $\phi(x, \lambda)$ интегральным преобразованием, аналогичным (10).

Сделав в уравнении $(22)$ подстановку $\Phi(w)=H(w)\left(1-w^{2}\right)^{-\frac{1}{2} \frac{r+2}{r+1}}$, а затем замену переменной $\tilde{\Lambda}(x)=H(w)$, где $x=\frac{1-w}{1+w}$, получим уравнение на функцию $\tilde{\Lambda}(x)$ :

$$
x^{\frac{r}{2(r+1)}}(1-x)^{\frac{r}{r+1}} \Lambda^{\prime}(x)=\nu \int_{x}^{1}(1-x / u)^{-\frac{r}{r+1}} u^{\frac{r-4}{2(r+1)}}(1-u)^{\frac{2-r}{r+1}} \Lambda(u) \frac{d u}{u} .
$$

И, наконец, после подстановки

$$
\tilde{\Lambda}(x)=x^{\frac{r+2}{2(r+1)}}(1-x)^{\frac{1}{r+1}} \Lambda(x)
$$

приходим к следуюшей спектральной задаче. Требуется найти все значения параметра $\nu$, при каждом из которых уравнение

$$
x(1-x) \Lambda^{\prime}(x)+\frac{(r+2)-(r+4) x}{2(r+1)} \Lambda(x)=\nu \int_{x}^{1}(u-x)^{-\frac{2}{r+1}}(1-u)^{\frac{3-r}{r+1}} \Lambda(u) d u
$$

имеет своим решением не равную тождественно нулю функцию $\Lambda(x)$, регулярную в единичном круге.

Отметим, что уравнение (23) вполне аналогично уравнению (17) и сводится к алгебраическому так же, как описано выше. 


\section{2. АНГАРМОНИЧЕСКИЙ ОСЦИЛЛЯТОР \\ С ПОТЕНЦИАЛОМ ВИДА $\left(x^{2}-\varepsilon\right)^{2}$}

Во введении мы отмечали, что потенциал вида $\left(x^{2}-\varepsilon\right)^{2}$ может описьвать две физически различные ситуации: $\varepsilon>0$ отвечает нарушенной, а $\varepsilon \leq 0$ - восстановленной симметрии системы. Для изучения спектра уравнения Шредингера с потенциалом заданного типа можно использовать предложенную нами выше процедуру. Как и ранее, будем искать четные и нечетные решения уравнения

$$
\psi^{\prime \prime}-\left(x^{2}-\varepsilon\right)^{2} \psi=-\lambda \psi
$$

раздельно. Вначале ищем четные решения (24) в виде

$$
\psi(x)=\sum_{p=0}^{\infty} a_{p} \frac{x^{2 p}}{f_{p}}, \quad f_{p}=3^{2 p / 3} \frac{\Gamma\left(\frac{2 p+2}{3}\right)}{\Gamma(2 / 3)}, \quad p \in Z_{+} .
$$

Подставляя $(25)$ в $(24)$ и учитывая явный вид $f_{p}$, найдем рекуррентные соотношения

$$
(p+1) a_{p+1}+\varepsilon \sqrt[3]{3} \frac{\Gamma\left(\frac{2 p+1}{3}\right)}{\Gamma(2 p / 3)} a_{p-1}-(p-1) a_{p-2}=\left(\varepsilon^{2}-\lambda\right) \frac{\Gamma\left(\frac{2 p+1}{3}\right)}{\Gamma\left(\frac{2 p+2}{3}\right) 2 \sqrt[3]{3}} a_{p}
$$

После введения производящей функции

$$
\Phi(W)=\sum_{p=0}^{\infty} a_{p} W^{2 p / 3}
$$

и проведения некоторых несложных преобразований придем к уравнению

$$
\frac{3}{2} W^{1 / 3}\left\{\left(1-W^{2}\right) \Phi^{\prime}-\frac{2}{3} W \Phi\right\}+\frac{\varepsilon \sqrt[3]{3}}{\Gamma(2 / 3)}\left\{\frac{2}{3} W^{2 / 3} \Phi^{\#}+W^{5 / 3} \Phi^{\# \prime}\right\}=\frac{\left(\varepsilon^{2}-\lambda\right)}{2 \sqrt[3]{3} \Gamma(1 / 3)} \Phi^{*}
$$

здесь

$$
\begin{aligned}
& \Phi^{\#}(W)=\int_{0}^{1}(1-t)^{-1 / 3} \Phi(t W) d t, \\
& \Phi^{*}(W)=\int_{0}^{1}(1-t)^{-2 / 3} t^{-2 / 3} \Phi(t W) d t .
\end{aligned}
$$

Далее воспользуемся подстановками, аналогичными описанньм выше,

$$
x^{2 / 3} \tilde{\Lambda}^{\prime}(x)+b(1-x)^{2 / 3}\left[\tilde{\Lambda}^{\#}+3(1+x) \tilde{\Lambda}^{\# \prime}\right]=\mu \tilde{\Lambda}^{*},
$$


где

$$
\begin{gathered}
b=\varepsilon\left[2^{1 / 3} 3^{5 / 3} \Gamma(2 / 3)\right]^{-1}, \quad \mu=\left(\lambda-\varepsilon^{2}\right)\left[2^{2 / 3} 3^{4 / 3} \Gamma(1 / 3)\right]^{-1}, \\
\tilde{\Lambda}^{\#}(x)=\int_{x}^{1}\left(1-\frac{x}{u}\right)^{-1 / 3} u^{-1 / 3} \tilde{\Lambda}(u) \frac{d u}{1+u} \\
\tilde{\Lambda}^{*}(x)=\int_{x}^{1}\left(1-\frac{x}{u}\right)^{-2 / 3}(1-u)^{-2 / 3} \tilde{\Lambda}(u) \frac{d u}{u}
\end{gathered}
$$

В полном соответствии с изложенным в разделе 1 , т.е. с ситуацией ненарушенной симметрии, решение (26) ишется в виде

$$
\tilde{\Lambda}(x)=x^{1 / 3} \Lambda(x) .
$$

В итоге для четных решений получим уравнение

$$
x \Lambda^{\prime}+\frac{1}{3} \Lambda+b(1-x)^{2 / 3}\left[\Lambda^{\#}+3(1+x) \Lambda^{\# \prime}\right]=\mu \Lambda^{*} .
$$

Соответственно для нечетных решений найдем

$$
x(1-x) \Lambda^{\prime}+\left(\frac{2}{3}-x\right) \Lambda+b(1-x)^{2 / 3}\left[\Lambda_{1}^{\#}+3(1+x) \Lambda_{1}^{\# \prime}\right]=\mu \Lambda^{*} .
$$

Отметим, что функция $\Lambda(x)$ - решение (29) и (30) является регулярной в единичном круге. Функции $\Lambda^{\#}(x), \Lambda_{1}^{\#}(x)$ и $\Lambda^{*}(x)$, фигурируюшие в $(29)$ и $(30)$, определяются следуюшим образом:

$$
\begin{aligned}
\Lambda^{\#}(x) & =\int_{x}^{1}(u-x)^{-1 / 3} \Lambda(u) \frac{d u}{1+u}, \\
\Lambda_{1}^{\#}(x) & =\int_{x}^{1}(u-x)^{-1 / 3}(1-u) \Lambda(u) \frac{d u}{1+u}, \\
\Lambda^{*}(x) & =\int_{x}^{1}(u-x)^{-2 / 3}(1-u)^{-2 / 3} \Lambda(u) \frac{d u}{u} .
\end{aligned}
$$

Следует указать, что при $\varepsilon \rightarrow 0 \quad(29)$ и (30) переходят в соответствуюшие уравнения для потенциала с вырождением $x^{4}$. Сведение задачи к алгебраической осуществляется приемом, описанным вьше. Дальнейшее решение задачи о нахождении спектра (даже в приближенном виде) указывает на сложную зависимость собственных значений от параметра $\varepsilon$, и получение ее в замкнутом виде затруднительно. Вместе с тем грубая оценка для основного состояния потенциала $x^{4}$, основанная на решении кубических уравнений (для четных и нечетных уровней), осушествляемая без использования компьютера, дает результат $\lambda_{0}=1.089$, что отличается от найденного нами (с точностью до десяти 
знаков) по процедуре, описанной ниже, следуюшего значения $\lambda_{0}=1.060352090$ на величину 0.03 , т.е. порядка $3 \%$. Последнее обстоятельство позволяет надеяться на возможное использование для основного уровня энергии уравнения Шредингера с нарушенной симметрией соответствуюшего выражения, аналитически учитывающего зависимость от параметра $\varepsilon$.

\section{3. ЧИСЛЕННОЕ ИССЛЕДОВАНИЕ СПЕКТРА УРАВНЕНИЯ ШРЕДИНГЕРА ДЛЯ ПОТЕНЦИАЛОВ $x^{2 r}(r=2,3, \ldots, 18)$}

Как было показано нами выше, вопрос о нахож дении спектра уравнения Шредингера для потенциала $x^{2 r}$ в точке вырождения может быть сведен к решению алгебраической задачи.

Для того чтобы не искать явное выражение для этой задачи, вновь запишем ее в виде, удобном для проведения численных расчетов и учитываюшем четности решений, а также компактную форму записи констант,

$$
(n+\beta) a_{n}+(1-n+\alpha) a_{n-1}=\nu B\left(\frac{r-1}{r+1}, \frac{4}{r+1}\right) \frac{\left(\frac{2}{r+1}\right)_{n}}{n !} \sum_{m=0}^{\infty} a_{m} b_{m-n}
$$

где

$$
\nu=\lambda(r+1)^{-2 r /(r+1)} 2^{-2 /(r+1)} / \Gamma\left(\frac{r-1}{r+1}\right),
$$

а значения $\alpha$ и $\beta$ для четных и нечетных решений следует положить равными

$$
\alpha=\left\{\begin{array}{ll}
-\frac{r+6}{2(r+1)} & \text { для четных, } \\
-\frac{r+4}{2(r+1)} & \text { для нечетных, }
\end{array} \quad \beta= \begin{cases}\frac{r}{2(r+1)} & \text { для четных, } \\
\frac{r+2}{2(r+1)} & \text { для нечетных, }\end{cases}\right.
$$

соответственно имеем

$$
b_{k}= \begin{cases}\frac{\Gamma\left(k+\frac{r-1}{r+1}\right) \Gamma\left(\frac{r+3}{r+1}\right)}{\Gamma\left(\frac{r-1}{r+1}\right) \Gamma\left(k+\frac{r+3}{r+1}\right)} & \text { при } k>0, \\ 1 & \text { при } k=0, \\ \frac{\Gamma\left(k-\frac{2}{r+1}\right) \Gamma\left(\frac{2}{r+1}\right)}{\Gamma\left(-\frac{2}{r+1}\right) \Gamma\left(k+\frac{2}{r+1}\right)} & \text { при } k<0 .\end{cases}
$$

Мы видим, что несмотря на то что такая алгебраическая задача также оказывается не решаемой точно, все же имеется возможность построить ее приближенное решение как для спектра, так и для собственных значений. Более точно, бесконечные вектор и матрица в (32) могут быть приближены посредством использования процедуры конечномерной аппроксимации, т.е. индекс $n$ будет пробегать значения до некоторых конечных величин $N_{1}, N_{2}, \ldots, N_{k}$. Такая возможность обусловливается тем, что, как отмечалось 


\begin{tabular}{|c|c|c|c|}
\hline № & $x^{4}$ & $x^{6}$ & $x^{14}$ \\
\hline 0 & 1.060352090 & 1.144892454 & 1.421438884 \\
\hline 1 & 3.799673030 & 4.338598712 & 5.636185031 \\
\hline 2 & 7.455697938 & 9.073084561 & 12.51210199 \\
\hline 3 & 11.64474551 & 14.93516963 & 21.87477520 \\
\hline 4 & 16.26182602 & 21.71416542 & 33.54989074 \\
\hline 5 & 21.23837292 & 29.29964594 & 47.38927498 \\
\hline$\vdots$ & & & \\
\hline 27 & 181.38226662 & 326.6885012 & 783.8625859 \\
\hline 28 & 190.2292387 & 344.6661934 & 834.3977651 \\
\hline 29 & 199.1799188 & 362.9621614 & 886.2808594 \\
\hline
\end{tabular}

выше, операторы в (32) компактные и могут быть аппроксимированыпоследовательностью конечномерных операторов [24]. При такой аппроксимации мы будем воспроизводить соответствующее число собственных значений приближаемого оператора, причем $\left\{\lambda_{0}^{\left(N_{i}\right)}, \ldots, \lambda_{N_{i}}^{\left(N_{i}\right)}\right\} \underset{N_{i} \rightarrow \infty}{\longrightarrow}\left\{\lambda_{0}, \ldots, \lambda_{N_{i}}\right\}$.

Сделаем несколько замечаний относительно предложенной вычислительной схемы. Во-первых, такая итерационная схема сходяшаяся. Во-вторых, она позволяет без обращения к ЭВМ вычислить приближенно первые четыре уровня энергии осциллятора, т.к. при этом мы должны решать алгебраическое уравнение четвертой степени на четвертом итерационном шаге. В-третьих, обратим внимание, что сам итерационный процесс организован следуюшим образом. На пятом шаге мы должны решать задачу о нахождении корней полинома пятой степени. Напомним, что на предыдушем шаге мы уже приближенно вычислили четыре корня. Таким образом, приближенно оценить границы пятого корня с учетом того факта, что последовательность собственных значений является монотонно возрастаюшей, не представляет особого труда. На шестом шаге идет уточнение предыдуших значений первых пяти корней и оцениваются границы расположения шестого. В качестве примера мы приведем результаты (которые совпадают с результатами работы [25]) вычислений спектра для потенциалов $x^{4}, x^{6}$ и $x^{14}$ (см. таблицу).

\section{ЗАКЛЮЧЕНИЕ}

В работе предложена регулярная аналитическая процедура нахождения спектра уравнения Шредингера с вырожденным потенциалом типа $x^{2 r}[26]$. Эта процедура основана на сведении исходной задачи на собственные векторы и собственные значения дифференциального оператора к алгебраической. Показано, что при решении этой задачи можно реализовать несколько путей, предложенных в явном виде в настояшей ра- 
боте. Один из них - построение нового интегрального преобразования со специальным ядром (см. формулу (10) настоящей работы), для осушествления второго необходимо построить новые классы специальных функций, используя многочлены совместной ортогональности с соответствуюшими весами.

Авторы выражают благодарность А.В.Борисову, В.В.Владимирскому, П.В. Елютину, В. Ч. Жуковскому, Л.В.Келдьшу, В. Д. Муру, В. С. Попову и И. В. Тютину за полезные советы и обсуждение, а также Д.В. Перегудову за подготовку рукописи к печати. Авторы благодарят Российский фонд фундаментальных исследований (грант № 93-01-278), INTAS (грант № 93-219) за частичную финансовую поддержку данной работы.

Публикация данной работы частично поддержана Международным научным фондом Cороса (грант № MRU300 и N7N000) и Российским правительством.

\section{Список литературы}

[1] E. Schrödinger. Ann. Phys. 1926. V. 79. P. 361; 489.

[2] В. А. Фок. Начала квантовой механики. М.: Наука, 1976.

[3] В.Г. Багров, А. С. Виивцев, С. В. Кетов. Дополнительные главы математической физики (калибровочные поля). М.: Изд-во МИРЭА, 1990; В. Г. Багров, А. С. Виивчев. Простейшие применения алгебр симметрии к решению задач квантовой механики: Препринт № 31. Томск: Томский филиал СО АН СССР, 1986.

[4] О. Б. Заславский, В. В. Ульянов. ЖЭЭТФ. 1984. Т. 87. № 5. С. 1724-1733.

[5] M. A. Shifman, A. V. Turbiner. Quantal problems with partial algebraization of the spectrum: Preprint ITEP-174. Moscow: Atominform, 1988.

[6] A. Yu. Morozov, A. M. Perelomov, A. A. Rosly, M. A. Shifman, A. V. Turbiner Quasi-exactlysolvable quantal problems: one-dimensional analoque of rational conformal field theories: Preprint MIT.CPT \# 1741, 1989; A. V. Turbiner. Contemporary Math. 1990. V. 160. P. 263-310.

[7] А.Г. Ушверидзе. ЭЧАЯ. 1989. Т. 20. № 5. С. 1185-1245.

[8] А.С. Виивчев, В. Ч. Жуковский, Р. А. Потапов, А. О. Старинец. Изв. Вузов. Физика. 1993. T. 2. C. $76-88$.

[9] В. Н. Сорокин. Обобщение классических ортогональных многочленов и сходимость совместных аппроксимаций Паде. Тр. семинара им. Г.И. Петровского. М.: МГУ, 1986. Т. 11. C. $125-165$.

[10] А. С. Виивцев, В. Н. Сорокин. Изв. Вузов. Физика. 1994. Т. 1. С. 95-101.

[11] М. Рид, Б. Саймон. Методы современной математической физики. Т. 4. Анализ операторов. М.: Мир, 1982.

[12] Т. Като. Теория возмущений линейных операторов. М.: Мир, 1972.

[13] В.Е. Захаров, С.В. Манаков, С. П. Новиков, П. П. Питаевский. Теория солитонов: метод обратной задачи. М.: Наука, 1980.

[14] В. П. Маслов. Асимптотические методы и теория возмущений. М.: Наука, 1988.

[15] Н. Я. Виленкин. Специальные функции и теория представлений групп. М.: Наука, 1991.

[16] М. В. Федорюк. Асимптотические методы для линейных ОДУ. М.: Наука, 1983.

[17] В.Г. Багров, Д. М. Гитман, И. М. Тернов, В.Р. Халилов, В. Н. Шаповалов. Точные решения релятивистских волновых уравнений. Новосибирск: Наука, 1982.

[18] V. G. Bagrov, D. M. Gitman. Exact Solutions of Relativisic Wave Equations. London: Kluwer Academic Publishers Dordrecht (Boston), 1990. 
[19] Р. Гилмор. Прикладная теория катастроф. М.: Мир, 1984.

[20] Т. Постон, И. Стюарт. Теория катастрофи ие приложения. М.: Мир, 1980.

[21] В. И. Арнольд. Теория катастроф. М.: МГУ, 1983.

[22] Л.Д. Ландау, Е. М. Лифииц. Статистическая физика (Теоретическая физика, т. 5). М.: Наука, 1964.

[23] Г. Стенли. Фазовые переходы и критические явления. М.: Мир, 1973.

[24] A.Н. Колмогоров, С. В. Фомин. Элементы теории функций и функционального анализа. М.: Наука, 1972.

[25] A. Hautot, A. Magnus. J. Comp. Appl. Math. 1979. V. 5. № 1. P. 3-15.

[26] Л. Д. Ландау, Е. М. Лифииц. Квантовая механика. Нерелятивистская теория (Теоретическая физика, т. 3). М.: Наука, 1989.

Поступила в редакцию 8.VIII.1995 г.

\section{A.S. Vshivzev, N. V. Norin, V.N. Sorokin \\ SOLUTION OF SPECTRAL PROBLEM FOR SCHRÖDINGER EQUATION WITH DEGENERATE POLINOMIAL POTENTIAL OF EVEN POWER}

The symmetry of the stationary Schrödinger equation with a degenerate potential $U(x)=x^{2 r}$, $r \in Z_{+}$, describing phase transitions in quantum systems, is reveled. The analytical procedure of finding the eigenvalues of the potentials in question is constructed and realized numerically for $r=$ $2,3, \ldots, 18$. The low energy levels are found. 